\title{
Convolutions of Harmonic Mappings Convex in the Horizontal Direction
}

\author{
Serkan Çakmak (D), Elif Yaşar $(\mathbb{D}$, and Sibel Yalçın \\ Department of Mathematics, Faculty of Arts and Science, Bursa Uludag University, 16059 Bursa, Turkey \\ Correspondence should be addressed to Elif Yaşar; elifyasar@uludag.edu.tr
}

Received 8 June 2021; Accepted 2 August 2021; Published 6 September 2021

Academic Editor: Humberto Rafeiro

Copyright $\odot 2021$ Serkan Çakmak et al. This is an open access article distributed under the Creative Commons Attribution License, which permits unrestricted use, distribution, and reproduction in any medium, provided the original work is properly cited.

In this paper, we establish some results concerning the convolutions of harmonic mappings convex in the horizontal direction with harmonic vertical strip mappings. Furthermore, we provide examples illustrated graphically with the help of Maple to illuminate the results.

\section{Introduction}

For real-valued harmonic functions $u$ and $v$ in the open unit disk $\mathbb{E}=\{z \in \mathbb{C}:|z|<1\}$, the complex-valued continuous function $f=u+i v$ is said to be harmonic and can be expressed as $f=h+\bar{g}$, where $h$ and $g$ are analytic in $\mathbb{E}$. Let $H$ be the class of harmonic mappings $f=h+\bar{g}$ normalized by $h(0)=g(0)=h^{\prime}(0)-1=0$ and have the following power series representations:

$$
\begin{aligned}
& h(z)=z+\sum_{m=2}^{\infty} a_{m} z^{m}, \\
& g(z)=\sum_{m=1}^{\infty} b_{m} z^{m} .
\end{aligned}
$$

We call $h$ the analytic part and $g$ the coanalytic part of $f$, respectively. The Jacobian of $f=h+\bar{g}$ is given by $J_{f}=\left|h^{\prime}\right|^{2}$ $-\left|g^{\prime}\right|^{2}$. Lewy's theorem [1] implies that $f \in H$ is locally univalent and sense-preserving if and only if $J_{f}>0$ in $\mathbb{E}$. The condition $J_{f}>0$ is equivalent to that dilatation $\omega(z)=g^{\prime}(z)$ $/ h^{\prime}(z)$ satisfying $|\omega(z)|<1$ for all $z \in \mathbb{E}$ (see $\left.[2,3]\right)$.

We denote by $\mathbb{S}_{H}$ the class of all harmonic, sense-preserving, and univalent mappings $f=h+\bar{g}$ in $\mathbb{E}$, which are normalized by the condition $h(0)=g(0)=0$ and $h^{\prime}(0)=1$.
Let $\mathbb{S}_{H}^{0}$ be the subset of all $f \in \mathbb{S}_{H}$ in which $g^{\prime}(0)=0$. Further, let $\mathbb{K}_{H}, \mathbb{C}_{H}$ (resp., $\mathbb{K}_{H}^{0}, \mathbb{C}_{H}^{0}$ ) be the subset of $\mathbb{S}_{H}$ (resp., $\mathbb{S}_{H}^{0}$ ) whose images are convex and close-to-convex domains. A domain $\Omega$ is said to be convex in the horizontal direction (CHD) if the intersection of $\Omega$ with each horizontal line is connected (or empty). A function $f=h+\bar{g} \in \mathbb{S}_{H}$ is said to be a CHD mapping if $f$ maps $\mathbb{E}$ onto a CHD domain. Let $\mathbb{S}_{\mathrm{CHD}}$ be the subset of $\mathbb{C}_{H}$ which consist of CHD mappings. The following basic theorem of Clunie and Sheil-Small [2] is known as shear construction that constructs harmonic mappings with prescribed dilatations onto a domain convex in one direction.

Theorem 1 (see [2]). A locally univalent harmonic mapping $f=h+\bar{g}$ in $\mathbb{E}$ is a univalent mapping of $\mathbb{E}$ onto a domain convex in a direction $\phi$ if and only if $h-e^{2 i \phi} g$ is a conformal univalent mapping of $\mathbb{E}$ onto a domain convex in the direction of $\phi$.

Let $f * F=h * H+\overline{g * G}$ be the convolution of two harmonic functions $f=h+\bar{g}$ and $F=H+\bar{G}$ where the operator $*$ is convolution (or Hadamard product) of two power series.

There are several research papers in recent years which investigate the convolution of harmonic univalent functions. In particular, Dorff [4] and Dorff et al. [5] studied the convolution of harmonic univalent mappings in the right half- 
plane. For some recent investigations involving convolution of harmonic mappings, we refer the reader to [6-13].

Let $F_{\mathfrak{a}}=H_{\mathfrak{a}}+\overline{G_{\mathfrak{a}}}$ sheared by $H_{\mathfrak{a}}-G_{\mathfrak{a}}=z /(1-z)$ with the dilatation $\omega_{\mathfrak{a}}=(\mathfrak{a}+z) /(1+\mathfrak{a} z)$, where $\mathfrak{a} \in(-1,1)$. Using shear construction of Clunie and Sheil-Small [2], we have

$$
\begin{gathered}
H_{\mathfrak{a}}(z)=\frac{1 /(1-\mathfrak{a}) z-1 / 2 z^{2}}{(1-z)^{2}}=\frac{1}{2}\left[\frac{z}{1-z}+\frac{1+\mathfrak{a}}{1-\mathfrak{a}} \frac{z}{(1-z)^{2}}\right] \\
G_{\mathfrak{a}}(z)=\frac{\mathfrak{a} /(1-\mathfrak{a}) z+1 / 2 z^{2}}{(1-z)^{2}}=\frac{1}{2}\left[\frac{-z}{1-z}+\frac{1+\mathfrak{a}}{1-\mathfrak{a}} \frac{z}{(1-z)^{2}}\right]
\end{gathered}
$$

It is clear that by setting $\mathfrak{a}=0$ in (2) and (3), we obtain $F_{0}=H_{0}+\overline{G_{0}}$ which satisfy the conditions $H_{0}-G_{0}=z /(1-$ $z$ ) and $\omega(z)=z$, studied by Liu and Li [8]. Wang et al. [14] also studied convolutions of this mapping. Note that $F_{\mathfrak{a}}$ is a CHD mapping.

Recently, Liu and Li [8] introduced the following generalized harmonic univalent mappings:

$$
\begin{aligned}
P_{\delta}(z)= & H_{\delta}(z)+\overline{G_{\delta}(z)}=\frac{1}{1+\delta}\left[\frac{\delta z}{(1-z)^{2}}+\frac{z}{1-z}\right] \\
& +\frac{1}{1+\delta} \overline{\left[\frac{\delta z}{(1 z)^{2}} \frac{z}{1 z}\right]}
\end{aligned}
$$

where $\delta>0$ and $z \in \mathbb{E}$. Obviously, $P_{1}(z)=F_{0}(z)$. If $f=h+\bar{g}$ $\in \mathbb{S}_{H}$, then

$$
P_{\delta} * f=\frac{\delta z h^{\prime}+h}{1+\delta}+\frac{\overline{\delta z g^{\prime} g}}{1+\delta}
$$

Also, $P_{\delta}(z)$ maps $\mathbb{E}$ onto the domain $\left\{u+i v: v^{2}>-[(2 \delta\right.$ )$\left.\left./(1+\delta) u+\left(1 /(1+\delta)^{2}\right)\right], \delta>0\right\}$ which is a CHD domain. Very recently, Yasar and Ozdemir [15] studied convolutions of these generalized harmonic mappings.

Let $f_{\gamma}=h_{\gamma}+\overline{g_{\gamma}} \in \mathbb{S}_{\mathrm{CHD}}^{0}$ with

$$
h_{\gamma}-g_{\gamma}=\frac{1}{2 i \sin \gamma} \log \left(\frac{1+z e^{i \gamma}}{1+z e^{-i \gamma}}\right) \text {, }
$$

where $\pi / 2 \leq \gamma<\pi$.

In this paper, we investigate the conditions under which the convolutions of harmonic mappings $P_{\delta}, f_{\gamma}$, and $F_{a}$ with prescribed dilatations are univalent and CHD provided that the convolutions are locally univalent and sense-preserving.
Furthermore, we provide two examples illustrated graphically with the help of Maple to illuminate our results.

\section{Preliminary Results}

Lemma 2 (see [16]). Let $f$ be an analytic fuction in $\mathbb{E}$ with $f$ $(0)=0$ and $f^{\prime}(0) \neq 0$ and let

$$
\varphi(z)=\frac{z}{\left(1+z e^{i \theta_{1}}\right)\left(1+z e^{i \theta_{2}}\right)},
$$

where $\theta_{1}, \theta_{2} \in \mathbb{R}$. If

$$
\operatorname{Re}\left(\frac{z f^{\prime}(z)}{\varphi(z)}\right)>0
$$

then $f$ is convex in the horizontal direction.

Lemma 3 (see [17]). Let $\varphi$ and $G$ be analytic in $\mathbb{E}$ with $\varphi^{\prime}(0)$ $=G(0)=0$. If $\varphi$ is convex and $G$ is starlike, then for each function $F$ analytic in $\mathbb{E}$ and satisying $\operatorname{Re}(F(z))>0$, we have

$$
\operatorname{Re}\left(\frac{(\varphi * F G)(z)}{(\varphi * G)(z)}\right)>0(z \in \mathbb{E}) .
$$

Lemma 4 ([18], Cohn's rule). Given a polynomial

$$
p(z)=p_{0}(z)=a_{k, 0} z^{k}+a_{k-1,0} z^{k-1}+\cdots+a_{1,0} z+a_{0,0}\left(a_{k, 0} \neq 0\right)
$$

of degree $k$, let

$$
\begin{aligned}
p^{*}(z)= & p_{0}^{*}(z)=z^{k} \overline{p\left(\frac{1}{\bar{z}}\right)}=\overline{a_{k, 0}}+\overline{a_{k 1,0}} z+\cdots+\overline{a_{1,0}} z^{k-1} \\
& +\overline{a_{0,0}} z^{k}\left(a_{k, 0} \neq 0\right) .
\end{aligned}
$$

Denote by $r$ and $s$ the number of zeros of $p(z)$ inside the unit circle and on it, respectively. If $\left|a_{0,0}\right|<\left|a_{k, 0}\right|$, then

$$
p_{1}(z)=\frac{\overline{a_{k, 0}} p(z)-a_{0,0} p^{*}(z)}{z}
$$

is of degree $k-1$ with $r_{1}=r-1$ and $s_{1}=s$ the number of zeros of $p_{1}(z)$ inside the unit circle and on it, respectively.

Lemma 5. Let $P_{\delta}=H_{\delta}+\overline{G_{\delta}}$ be defined by (4) and $f_{\gamma}=h_{\gamma}+\overline{g_{\gamma}}$ be defined by (6) with dilatation $\omega=g_{\gamma}^{\prime} / h_{\gamma}^{\prime}$. Then the dilatation of $P_{\delta} * f_{\gamma}$ is given by

$$
\tilde{\omega}(z)=\frac{\omega(1-\omega)\left[(\delta-1)-2 z \cos \gamma-(\delta+1) z^{2}\right]+\delta z \omega^{\prime}\left(1+2 z \cos \gamma+z^{2}\right)}{(1-\omega)\left[(\delta+1)+2 z \cos \gamma-(\delta-1) z^{2}\right]+\delta z \omega^{\prime}\left(1+2 z \cos \gamma+z^{2}\right)}
$$


Proof. Since $h_{\gamma}-g_{\gamma}=1 /(2 i \sin \gamma) \log \left(\left(1+z e^{i \gamma}\right) /\left(1+z e^{-i \gamma}\right)\right)$ $(\pi / 2 \leq \gamma<\pi)$ and $g_{\gamma}^{\prime}=\omega h_{\gamma}^{\prime}$, then $g_{\gamma}^{\prime}=\omega^{\prime} h_{\gamma}^{\prime}+\omega h_{\gamma}^{\prime}$. We immediately get

$$
h_{\gamma}^{\prime}=\frac{1}{(1-\omega)\left(1+z e^{i \gamma}\right)\left(1+z e^{-i \gamma}\right)},
$$

$$
h_{\gamma}^{\prime}=-\frac{2(\cos \gamma+z)(1-\omega)-\omega^{\prime}\left(1+2 z \cos \gamma+z^{2}\right)}{(1-\omega)^{2}\left(1+z e^{i \gamma}\right)^{2}\left(1+z e^{-i \gamma}\right)^{2}} .
$$

From (4), we have

$$
\begin{aligned}
\tilde{\omega}(z) & =\frac{\left(G_{\delta} * g_{\gamma}\right)^{\prime}}{\left(H_{\delta} * h_{\gamma}\right)^{\prime}}=\frac{\left(\delta z g_{\gamma}^{\prime}-g_{\gamma}\right)^{\prime}}{\left(\delta z h_{\gamma}^{\prime}+h_{\gamma}\right)^{\prime}}=\frac{(\delta-1) g_{\gamma}^{\prime}+\delta z g_{\gamma}^{\prime}}{(\delta+1) h_{\gamma}^{\prime}+\delta z h_{\gamma}^{\prime}}=\frac{(\delta-1) \omega h_{\gamma}^{\prime}+\delta z\left(\omega^{\prime} h_{\gamma}^{\prime}+\omega h_{\gamma}^{\prime}\right)}{(\delta+1) h_{\gamma}^{\prime}+\delta z h_{\gamma}^{\prime}} \\
& =\frac{\omega(1-\omega)\left[(\delta-1)-2 z \cos \gamma-(\delta+1) z^{2}\right]+\delta z \omega^{\prime}\left(1+2 z \cos \gamma+z^{2}\right)}{(1-\omega)\left[(\delta+1)+2 z \cos \gamma-(\delta-1) z^{2}\right]+\delta z \omega^{\prime}\left(1+2 z \cos \gamma+z^{2}\right)} .
\end{aligned}
$$

Lemma 6. Let $P_{\delta}=H_{\delta}+\overline{G_{\delta}}$ be defined by (4) and $f_{\gamma}=h_{\gamma}+\overline{g_{\gamma}}$ be defined by (6). If $P_{\delta} * f_{\gamma}$ is locally univalent and sense-preserving, then $P_{\delta} * f_{\gamma}$ is univalent and convex in the horizontal direction.

Proof. Let

$$
\begin{aligned}
F_{1} & =\left(H_{\delta}-G_{\delta}\right) *\left(h_{\gamma}+g_{\gamma}\right) \\
& =H_{\delta} * h_{\gamma}+H_{\delta} * g_{\gamma}-G_{\delta} * h_{\gamma}-G_{\delta} * g_{\gamma}, \\
F_{2} & =\left(H_{\delta}+G_{\delta}\right) *\left(h_{\gamma}-g_{\gamma}\right) \\
& =H_{\delta} * h_{\gamma}-H_{\delta} * g_{\gamma}+G_{\delta} * h_{\gamma}-G_{\delta} * g_{\gamma} .
\end{aligned}
$$

Thus,

$$
H_{\delta} * h_{\gamma}-G_{\delta} * g_{\gamma}=\frac{1}{2}\left(F_{1}+F_{2}\right)
$$

By Theorem 1 , we need to prove that $1 / 2\left(F_{1}+F_{2}\right)$ is convex in the horizontal direction. Since

$$
h_{\gamma}-g_{\gamma}=\frac{1}{2 i \sin \gamma} \log \left(\frac{1+z e^{i \gamma}}{1+z e^{-i \gamma}}\right)\left(\frac{\pi}{2} \leq \gamma<\pi\right),
$$

we have

$$
\begin{aligned}
z F_{1}^{\prime} & =\left(H_{\delta}-G_{\delta}\right) *\left[z\left(h_{\gamma}^{\prime}+g_{\gamma}^{\prime}\right)\right] \\
& =\left(H_{\delta}-G_{\delta}\right) *\left[z\left(h_{\gamma}^{\prime}-g_{\gamma}^{\prime}\right)\left(\frac{h_{\gamma}^{\prime}+g_{\gamma}^{\prime}}{h_{\gamma}^{\prime}-g_{\gamma}^{\prime}}\right)\right] \\
& =\frac{2 z}{(1+\delta)(1-z)} * \frac{z}{\left(1+z e^{i \gamma}\right)\left(1+z e^{-i \gamma}\right)}\left(\frac{1+\omega_{\gamma}}{1-\omega_{\gamma}}\right) \\
& =\frac{2 z p_{1}(z)}{(1+\delta)\left(1+z e^{i \gamma}\right)\left(1+z e^{-i \gamma}\right)},
\end{aligned}
$$

where $p_{1}(z)=\left(1+\omega_{\gamma}\right) /\left(1-\omega_{\gamma}\right)$ satisfies the condition Re $\left(p_{1}(z)\right)>0$. Thus, we have

$$
\operatorname{Re}\left\{\frac{z F_{1}^{\prime}}{2 z /\left[(1+\delta)\left(1+z e^{i \gamma}\right)\left(1+z e^{-i \gamma}\right)\right]}\right\}=\operatorname{Re}\left\{p_{1}(z)\right\}>0 .
$$

Now, we consider

$$
\begin{aligned}
z F_{2}^{\prime} & =\left[z\left(H_{\delta}^{\prime}+G_{\delta}^{\prime}\right) *\left(h_{\gamma}-g_{\gamma}\right)\right] \\
& =\left[z\left(H_{\delta}^{\prime}-G_{\delta}^{\prime}\right) \frac{H_{\delta}^{\prime}+G_{\delta}^{\prime}}{H_{\delta}^{\prime}-G_{\delta}^{\prime}}\right] *\left(h_{\gamma}-g_{\gamma}\right) \\
& =\left[z\left(H_{\delta}^{\prime}-G_{\delta}^{\prime}\right)\left(\frac{1+\omega_{\delta}}{1-\omega_{\delta}}\right)\right] *\left(h_{\gamma}-g_{\gamma}\right) \\
& =\frac{2 z p_{2}(z)}{(1+\delta)(1-z)^{2}} *\left(h_{\gamma}-g_{\gamma}\right),
\end{aligned}
$$

where $p_{2}(z)=\left(1+\omega_{\delta}\right) /\left(1-\omega_{\delta}\right)$ satisfies the condition $\operatorname{Re}$ $\left\{p_{2}(z)\right\}>0$. Using the fact that

$$
\psi(z) * \frac{z}{(1-z)^{2}}=z \psi^{\prime}(z)
$$

and $h_{\gamma}-g_{\gamma}$ is convex, by Lemma 3 , we have

$$
\begin{aligned}
& \operatorname{Re}\left\{\frac{z F_{2}^{\prime}}{z /\left[\left(1+z e^{i \gamma}\right)\left(1+z e^{-i \gamma}\right)\right]}\right\} \\
& \quad=\operatorname{Re}\left\{\frac{\left(h_{\gamma}-g_{\gamma}\right) * p_{2}(z)\left[2 z /\left((1+\delta)(1-z)^{2}\right)\right]}{z\left(h_{\gamma}^{\prime}-g_{\gamma}^{\prime}\right)}\right\} \\
& =\operatorname{Re}\left\{\frac{\left(h_{\gamma}-g_{\gamma}\right) * p_{2}(z)\left[2 z /\left((1+\delta)(1-z)^{2}\right)\right]}{\left(h_{\gamma}-g_{\gamma}\right) * z /(1-z)^{2}}\right\}>0 .
\end{aligned}
$$


Finally, using Lemma 2, we obtain that $F_{1}+F_{2}$ is convex in the horizontal direction.
Lemma 7. Let $f_{\gamma}=h_{\gamma}+g_{\gamma} \in \mathbb{S}_{\text {CHD }}^{0}$ be given by (6) with dilatation $\omega=g_{\gamma}^{\prime} / h_{\gamma}^{\prime}$ and $F_{\mathfrak{a}}=H_{\mathfrak{a}}+\overline{G_{\mathfrak{a}}}$ be a mapping defined by (2) and (3). Then the dilatation of $F_{\mathfrak{a}} * f_{\gamma}$ is given by

$$
\tilde{W}(z)=\frac{2 \omega(1-\omega)\left[\mathfrak{a}-(1-\mathfrak{a}) z \cos \gamma-z^{2}\right]+(1+\mathfrak{a}) z \omega^{\prime}\left(1+2 z \cos \gamma+z^{2}\right)}{2(1-\omega)\left[1+(1-\mathfrak{a}) z \cos \gamma-\mathfrak{a} z^{2}\right]+(1+\mathfrak{a}) z \omega^{\prime}\left(1+2 z \cos \gamma+z^{2}\right)}
$$

Proof. From (2) and (3), we have

$$
\tilde{W}(z)=\frac{\left(G_{\mathfrak{a}} * g_{\gamma}\right)^{\prime}}{\left(H_{\mathfrak{a}} * h_{\gamma}\right)^{\prime}}=\frac{\left((1+\mathfrak{a}) z g_{\gamma}^{\prime}-(1-\mathfrak{a}) g_{\gamma}\right)^{\prime}}{\left((1+\mathfrak{a}) z h_{\gamma}^{\prime}+(1-\mathfrak{a}) h_{\gamma}\right)^{\prime}}
$$$$
=\frac{2 \mathfrak{a} g_{\gamma}^{\prime}+(1+\mathfrak{a}) z g_{\gamma}^{\prime}}{2 h_{\gamma}^{\prime}+(1+\mathfrak{a}) z h_{\gamma}^{\prime}}=\frac{2 \mathfrak{a} \omega h_{\gamma}^{\prime}+(1+\mathfrak{a}) z\left(\omega^{\prime} h_{\gamma}^{\prime}+\omega h_{\gamma}^{\prime}\right)}{2 h_{\gamma}^{\prime}+(1+\mathfrak{a}) z h_{\gamma}^{\prime}}
$$

Using (14) and (15), then we obtain the dilatation of $F_{\mathfrak{a}}$ $* f_{\gamma}$ as follows:

$$
\tilde{W}(z)=\frac{2 \omega(1-\omega)\left[\mathfrak{a}-(1-\mathfrak{a}) z \cos \gamma-z^{2}\right]+(1+\mathfrak{a}) z \omega^{\prime}\left(1+2 z \cos \gamma+z^{2}\right)}{2(1-\omega)\left[1+(1-\mathfrak{a}) z \cos \gamma-\mathfrak{a} z^{2}\right]+(1+\mathfrak{a}) z \omega^{\prime}\left(1+2 z \cos \gamma+z^{2}\right)} .
$$

Lemma 8 ([14], Lemma 2.4). Let $F_{\mathfrak{a}}=H_{\mathfrak{a}}+\overline{G_{\mathfrak{a}}}$ be a mapping defined by (2), (3) and $f_{\gamma}=h_{\gamma}+\overline{g_{\gamma}} \in \mathbb{S}_{C H D}^{0}$ be defined by (6). If $F_{\mathfrak{a}} * f_{\gamma}$ is locally univalent and sense-preserving, then $F_{\mathfrak{a}} * f_{\gamma}$ is univalent and convex in the horizontal direction.

Lemma 9 ([19], Gauss-Lucas theorem). Let $T(z)$ be a nonconstant polynomial with complex coefficients. Then, the zeros of the derivative $T^{\prime}(z)$ are contained in the convex hull of the set of the zeros of $T(z)$.

Lemma 10. Let

$$
\begin{aligned}
q(z)= & z^{k+1}+\frac{2(k+1) \cos \gamma}{k+2} z^{k}+\frac{k}{k+2} z^{k-1} \\
& -\frac{2}{k+2} e^{-i \theta} z-\frac{2 \cos \gamma}{k+2} e^{-i \theta}
\end{aligned}
$$

be a complex polynomial of degree $k+1$, where $\theta \in \mathbb{R}, k \in \mathbb{N}^{+}$, and $\pi / 2 \leq \gamma<\pi$. Then, all zeros of $q(z)$ lie in the closed unit disk $|z| \leq 1$.
Proof. Note that $q(z)=1 /(k+2) T^{\prime}(z)$, where

$$
T(z)=\left(z^{k}-e^{-i \theta}\right)\left(1+2 z \cos \gamma+z^{2}\right)
$$

It is obvious that the roots of $\left(z^{k}-e^{-i \theta}\right)$ lie on the unit circle. Also, $-\cos \gamma \pm i \sin \gamma$ which are the roots of $(1+2 z \cos$ $\gamma+z^{2}$ ) lie on the unit circle as well. Hence, the result follows from Lemma 9.

\section{Main Results}

Theorem 11. Let $P_{\delta}=H_{\delta}+\overline{G_{\delta}} \in \mathbb{S}_{C H D}$ be a mapping given by (4) and $f_{\gamma}=h_{\gamma}+\overline{g_{\gamma}} \in \mathbb{S}_{C H D}^{0}$ be given by (6) with the dilatation $\omega_{k}=g_{\gamma}^{\prime} / h_{\gamma}^{\prime}=e^{i \theta} z^{k}\left(\theta \in \mathbb{R}, k \in \mathbb{N}^{+}\right)$. Then $P_{\delta} * f_{\gamma}$ is univalent and convex in the horizontal direction.

Proof. By Lemma 6, we need to prove that the dilatation $\tilde{\omega}$ of $P_{\delta} * f_{\gamma}$ satisfies $|\tilde{\omega}|<1$ for all $z \in \mathbb{E}$. Substituting $\omega=e^{i \theta} z^{k}$ in (13), we yield

$$
\tilde{\omega}(z)=\frac{e^{i \theta} z^{k}\left(1-e^{i \theta} z^{k}\right)\left[(\delta-1)-2 z \cos \gamma-(\delta+1) z^{2}\right]+\delta k e^{i \theta} z^{k}\left(1+2 z \cos \gamma+z^{2}\right)}{\left(1-e^{i \theta} z^{k}\right)\left[(\delta+1)+2 z \cos \gamma-(\delta-1) z^{2}\right]+\delta k e^{i \theta} z^{k}\left(1+2 z \cos \gamma+z^{2}\right)}=e^{2 i \theta} z^{k} \frac{t(z)}{t^{*}(z)},
$$


where

$$
\begin{aligned}
t(z)= & z^{k+2}+\frac{2 \cos \gamma}{1+\delta} z^{k+1}+\frac{1-\delta}{1+\delta} z^{k} \\
& +\frac{\delta(k-1)-1}{1+\delta} e^{-i \theta} z^{2}+\frac{2(k \delta-1) \cos \gamma}{1+\delta} e^{-i \theta} z \\
& +\frac{\delta(1+k)-1}{1+\delta} e^{-i \theta}, \\
t^{*}(z)= & 1+\frac{2 \cos \gamma}{1+\delta} z+\frac{1-\delta}{1+\delta} z^{2}+\frac{\delta(k-1)-1}{1+\delta} e^{i \theta} z^{k} \\
& +\frac{2(k \delta-1) \cos \gamma}{1+\delta} e^{i \theta} z^{k+1}+\frac{\delta(1+k)-1}{1+\delta} e^{i \theta} z^{k+2} .
\end{aligned}
$$

If we substitute $\delta=2 / k$ into (30), then $t(z) / t^{*}(z)=e^{-i \theta}$, and it is clear that $|\tilde{\omega}|<1$ for all $z \in \mathbb{E}$. Now, we need to show that $|\tilde{\omega}|<1$ for $0<\delta<2 / k$. Obviously, if $z_{0}$ is a zero $t(z)$, then $1 / \overline{z_{0}}$ is zero of $t^{*}(z)$. Then, we may write

$$
\tilde{\omega}(z)=e^{2 i \theta} z^{k} \frac{\left(z+A_{1}\right)\left(z+A_{2}\right) \cdots\left(z+A_{k+2}\right)}{\left(1+\overline{A_{1}} z\right)\left(1+\overline{A_{2}} z\right) \cdots\left(1+\overline{A_{k+2}} z\right)} .
$$

Using Lemma 4, we only need to show that all zeros of (31) lie in the closed unit disk for $0<\delta<2 / k$. Since $\left|a_{0,0}\right|=\mid$ $(\delta(1+k)-1) /(1+\delta) e^{-i \theta}|=|(\delta(1+k)-1) /(1+\delta)|<| a_{k+2,0} \mid$ $=1$ for $0<\delta<2 / k$, thus we have

$$
\begin{aligned}
t_{1}(z)= & \frac{\overline{a_{k+2,0}} t(z)-a_{0,0} t^{*}(z)}{z}=\frac{\delta(k+2)(2-k \delta)}{(1+\delta)^{2}} \\
& \cdot\left(z^{k+1}+\frac{2(k+1) \cos \gamma}{k+2} z^{k}+\frac{k}{k+2} z^{k-1}\right. \\
& \left.-\frac{2}{k+2} e^{-i \theta} z-\frac{2 \cos \gamma}{k+2} e^{-i \theta}\right) .
\end{aligned}
$$

By Lemma 10, we know that all zeros of

$$
\begin{aligned}
q(z)= & z^{k+1}+\frac{2(k+1) \cos \gamma}{k+2} z^{k}+\frac{k}{k+2} z^{k-1} \\
& -\frac{2}{k+2} e^{-i \theta} z-\frac{2 \cos \gamma}{k+2} e^{-i \theta}
\end{aligned}
$$

lie inside the closed disk. Then, by Cohn's rule, $t(z)$ given by (31) has all its zeros in the closed unit disk. The proof is complete.

Theorem 12. Let $F_{\mathfrak{a}}$ be a mapping given by (2) and $f_{\gamma}=h_{\gamma}$ $+g_{\gamma} \in \mathbb{S}_{C H D}^{0}$ be a mapping given by (6) with the dilatation $\omega_{k}=g_{\gamma}^{\prime} / h_{\gamma}^{\prime}=e^{i \theta} z^{k}\left(\theta \in \mathbb{R}, k \in \mathbb{N}^{+}\right)$. Then, $F_{\mathfrak{a}} * f_{\gamma}$ is univalent and convex in the horizontal direction for $-1<\mathfrak{a} \leq(2-k) /$ $(2+k)$.

Proof. By Lemma 8, we need to prove that $F_{\mathfrak{a}} * f_{\gamma}$ is locally univalent and sense-preserving, i.e., the dilatation $\tilde{W}$ of $F_{\mathfrak{a}}$ $* f_{\gamma}$ satisfies $|\tilde{W}(z)|<1$ for all $z \in \mathbb{E}$. Substituting $\omega=e^{i \theta} z^{k}$ in (25),

$$
\begin{aligned}
\tilde{W}(z) & =e^{2 i \theta} z^{k} \times \frac{z^{k+2}+(1-\mathfrak{a}) \cos \gamma z^{k+1}-\mathfrak{a} z^{k}+((1+\mathfrak{a}) k-2) / 2 e^{-i \theta} z^{2}+[(k-1)+\mathfrak{a}(k+1)] \cos \gamma e^{-i \theta} z+((k+2) \mathfrak{a}+k) / 2 e^{-i \theta}}{1+(1-\mathfrak{a}) \cos \gamma z-\mathfrak{a} z^{2}+((1+\mathfrak{a}) k-2) / 2 e^{i \theta} z^{k}+[(k-1)+\mathfrak{a}(k+1)] \cos \gamma e^{i \theta} z^{k+1}+((k+2) \mathfrak{a}+k) / 2 e^{i \theta} z^{k+2}} \\
& =e^{2 i \theta} z^{k} \frac{u(z)}{u^{*}(z)},
\end{aligned}
$$

where

$$
\begin{aligned}
u(z)= & z^{k+2}+(1-\mathfrak{a}) \cos \gamma z^{k+1}-\mathfrak{a} z^{k}+((1+\mathfrak{a}) k-2) / 2 e^{-i \theta} z^{2} \\
& +[(k-1)+\mathfrak{a}(k+1)] \cos \gamma e^{-i \theta} z+((k+2) \mathfrak{a}+k) / 2 e^{-i \theta},
\end{aligned}
$$

$$
\begin{aligned}
u^{*}(z)= & 1+(1-\mathfrak{a}) \cos \gamma z-\mathfrak{a} z^{2}+((1+\mathfrak{a}) k-2) / 2 e^{i \theta} z^{k} \\
& +[(k-1)+\mathfrak{a}(k+1)] \cos \gamma e^{i \theta} z^{k+1} \\
& +((k+2) \mathfrak{a}+k) / 2 e^{i \theta} z^{k+2} .
\end{aligned}
$$

If we substitute $\mathfrak{a}=(2-k) /(2+k)$ into (36), we yield

$$
\tilde{W}(z)=e^{2 i \theta} z^{k} \frac{z^{k+2}+2 k /(k+2) \cos \gamma z^{k+1}-(2-k) /(k+2) z^{k}-(2-k) /(k+2) e^{-i \theta} z^{2}+2 k /(k+2) \cos \gamma e^{-i \theta} z+e^{-i \theta}}{1+2 k /(k+2) \cos \gamma z-(2-k) /(k+2) z^{2}-(2-k) /(k+2) e^{i \theta} z^{k}+2 k /(k+2) \cos \gamma e^{i \theta} z^{k+1}+e^{i \theta} z^{k+2}}=e^{i \theta} z^{k} .
$$




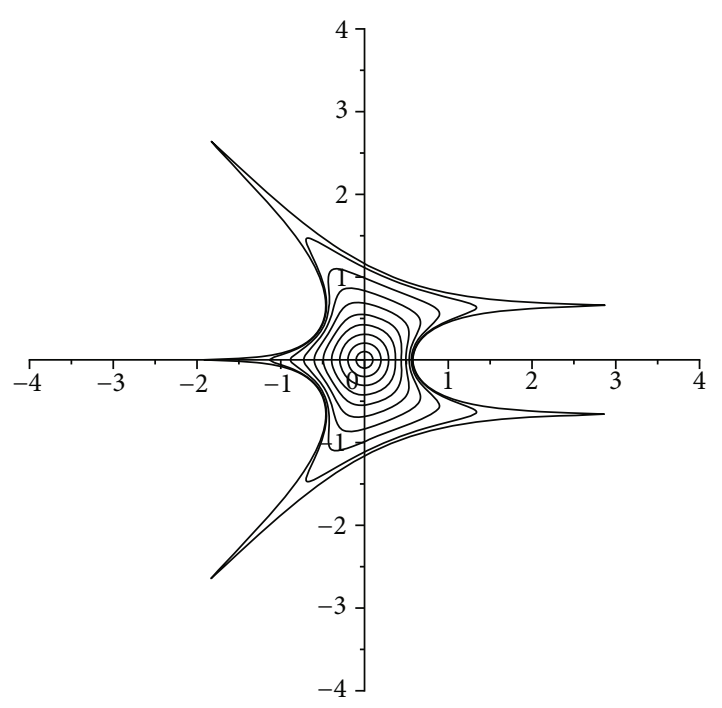

Figure 1: Image of $f_{\Pi / 2}$.

Hence, $|\tilde{W}(z)|=\left|e^{i \theta} z^{k}\right|<1$.

Next, we will show that $|\tilde{W}(z)|<1$ for all $-1<\mathfrak{a}<(2-$ $k) /(2+k)$. If $z_{0}$ is a zero of $u(z)$, then $1 / \overline{z_{0}}$ is zero of $u^{*}(z)$; hence,

$$
\begin{aligned}
\tilde{W}(z)= & e^{2 i \theta} z^{k} \frac{u(z)}{u^{*}(z)}=e^{2 i \theta} z^{k} \\
& \cdot \frac{\left(z+A_{1}\right)\left(z+A_{2}\right) \cdots\left(z+A_{k+2}\right)}{\left(1+\overline{A_{1}} z\right)\left(1+\overline{A_{2}} z\right) \cdots\left(1+\overline{A_{k+2}} z\right)} .
\end{aligned}
$$

By Lemma 4, we need to show that all zeros of $u(z)$ lie inside or on the unit disk for $-1<\mathfrak{a}<(2-k) /(2+k)$. Since

$$
\left|a_{0,0}\right|=\left|\frac{(k+2) \mathfrak{a}+k}{2} e^{-i \theta}\right|<1=\left|a_{k+2,0}\right| \text { for }-1<\mathfrak{a}<\frac{2-k}{2+k},
$$

from (12), we have

$$
\begin{aligned}
u_{1}(z)= & \frac{\overline{a_{k+2,0}} u(z)-a_{0,0} u^{*}(z)}{z} \\
= & -\frac{(k+2)(1+\mathfrak{a})[(k+2) \mathfrak{a}+k-2]}{4} \\
& \cdot\left(z^{k+1}+\frac{2(k+1) \cos \gamma}{k+2} z^{k}+\frac{k}{k+2} z^{k-1}\right. \\
& \left.-\frac{2}{k+2} e^{-i \theta} z^{k}-\frac{2 \cos \gamma}{k+2} e^{-i \theta}\right) \\
= & -\frac{(k+2)(1+\mathfrak{a})[(k+2) \mathfrak{a}+k-2]}{4} q(z),
\end{aligned}
$$

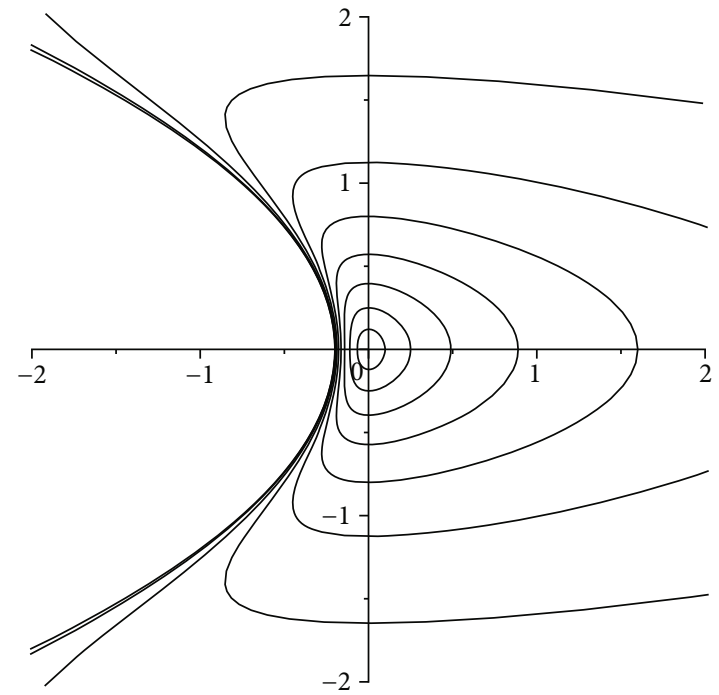

FIgURE 2: Image of $P_{2 / 3}$.

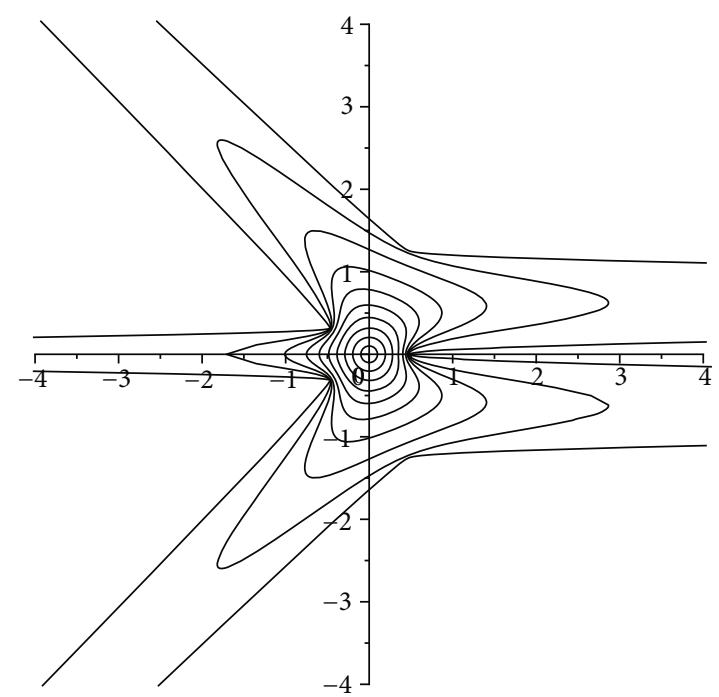

Figure 3: Image of $P_{2 / 3} * f_{\Pi / 2}$.

where

$$
\begin{aligned}
q(z)= & z^{k+1}+\frac{2(k+1) \cos \gamma}{k+2} z^{k}+\frac{k}{k+2} z^{k-1} \\
& -\frac{2}{k+2} e^{-i \theta} z-\frac{2 \cos \gamma}{k+2} e^{-i \theta} .
\end{aligned}
$$

Because $(k+2)(1+\mathfrak{a})[(k+2) \mathfrak{a}+k-2] / 4 \neq 0$ for $-1<\mathfrak{a}$ $<(2-k) /(2+k)$, it follows that both $u_{1}(z)$ and $q(z)$ have the same zeros. By Lemma 10, we know that all zeros of $q(z)$ lie inside the closed unit disk. Then, by Cohn's rule, we know that all zeros $u(z)$ lie inside or on the boundary of the unit disk. The proof is completed.

Theorem 13. Let $F_{0}=H_{0}+\overline{G_{0}} \in \mathbb{S}_{C H D}^{0}$ be a harmonic mapping with $H_{0}-G_{0}=z /(1-z)$ and dilatation $G_{0}^{\prime}(z) / H_{0}^{\prime}(z)=$ $z$. Let $f_{\pi / 2}=h_{\pi / 2}+\overline{g_{\pi / 2}} \in \mathbb{S}_{C H D}$ be a mapping defined by (6) 


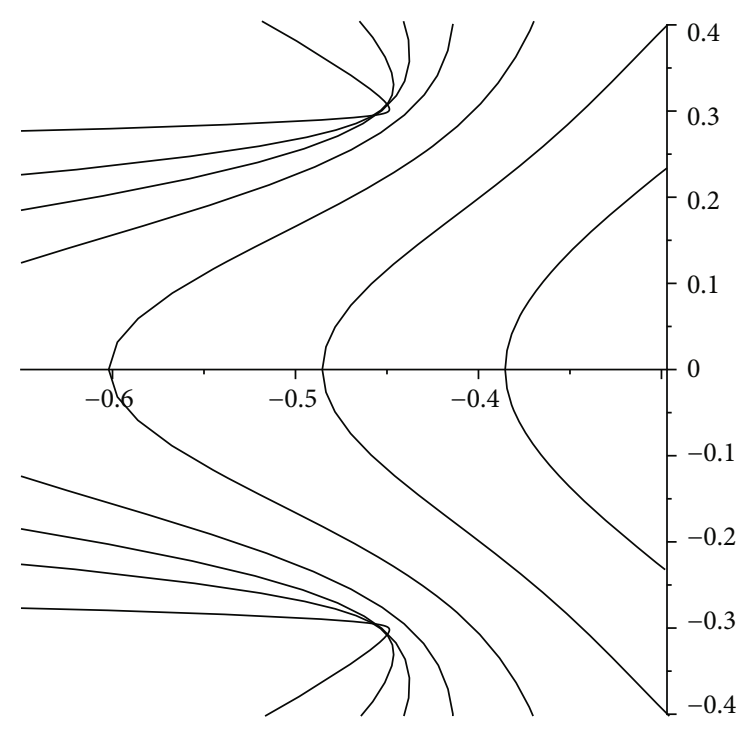

Figure 4: Image of $P_{3 / 4} * f_{\Pi / 2}$.

with $\gamma=\pi / 2$ and dilatation $\omega_{\mu}(z)=\left(\mu+z^{2}\right) /\left(1+\mu z^{2}\right)$, $-1<\mu<1$. Then the mapping $F_{0} * f_{\pi / 2}$ is univalent and convex in the horizontal direction.

Proof. Since $f_{\pi / 2}=h_{\pi / 2}+\overline{g_{\pi / 2}} \in \mathbb{S}_{\mathrm{CHD}}$ is a mapping defined by (6) with $\gamma=\pi / 2$, we have

$$
h_{\pi / 2}(z)-g_{\pi / 2}(z)=\frac{1}{2 i} \log \left(\frac{1+i z}{1-i z}\right)
$$

Therefore, we know that

$$
\begin{aligned}
\tilde{W}(z) & =\frac{\left(G_{0} * g_{\pi / 2}\right)^{\prime}}{\left(H_{0} * h_{\pi / 2}\right)^{\prime}}=\frac{\left(z g_{\pi / 2}^{\prime}-g_{\pi / 2}\right)^{\prime}}{\left(z h_{\pi / 2}^{\prime}+h_{\pi / 2}\right)^{\prime}} \\
& =\frac{z g_{\pi / 2}^{\prime}}{2 h_{\pi / 2}^{\prime}+z h_{\pi / 2}^{\prime}}=z \frac{\omega_{\mu} h_{\pi / 2}^{\prime}+\omega_{\mu}^{\prime} h_{\pi / 2}^{\prime}}{2 h_{\pi / 2}^{\prime}+z h_{\pi / 2}^{\prime}} .
\end{aligned}
$$

Substituting

$$
\begin{aligned}
& h_{\pi / 2}^{\prime}(z)=\frac{1}{\omega_{\mu}\left(1+z^{2}\right)}, \\
& h_{\pi / 2}^{\prime}(z)=\frac{\omega_{\mu}^{\prime}\left(1+z^{2}\right)-2 z \omega_{\mu}}{\left(1-\omega_{\mu}\right)^{2}\left(1+z^{2}\right)^{2}},
\end{aligned}
$$

into (44) yields

$$
\tilde{W}(z)=z \frac{\omega_{\mu}^{2}-\left(\omega_{\mu}-1 / 2 \omega_{\mu}^{\prime} z\right)+1 / 2 \omega_{\mu}^{\prime} 1 / z}{1 / z-\left(\omega_{\mu}-1 / 2 \omega_{\mu}^{\prime} z\right) 1 / z+1 / 2 \omega_{\mu}^{\prime} z^{2}} .
$$

Setting $\omega_{\mu}(z)=\left(\mu+z^{2}\right) /\left(1+\mu z^{2}\right)$ in the above equation, we get $\tilde{W}(z)=z^{2}$, and hence, $|\tilde{W}(z)|<1$ for all $z \in \mathbb{E}$.

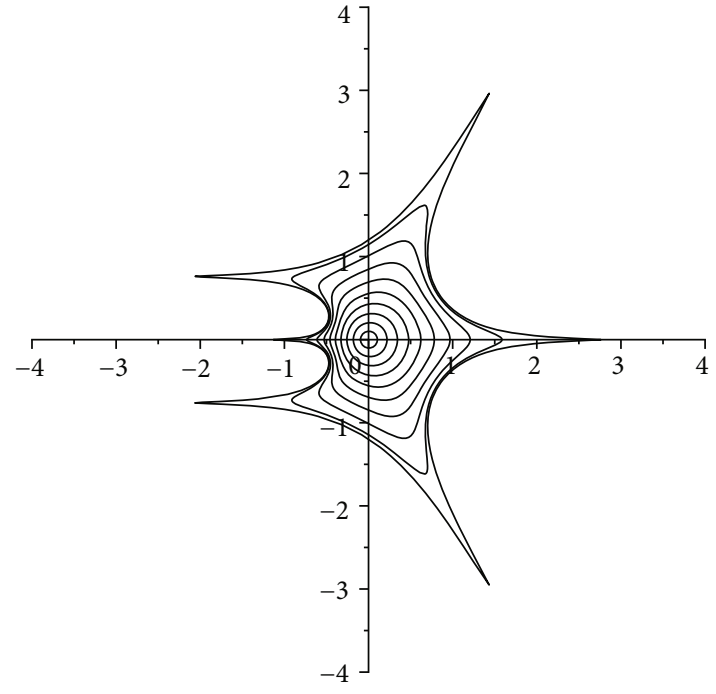

Figure 5: Image of $f_{2 \Pi / 3}$.

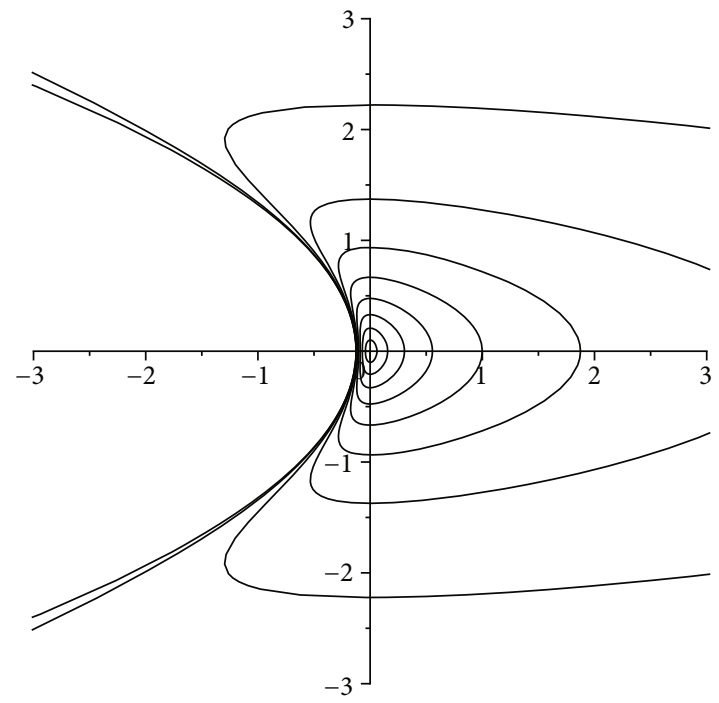

FIGURE 6: Image of $F_{-1 / 3}$.

Example 14. Suppose $f_{\gamma}=h_{\gamma}+\overline{g_{\gamma}} \in \mathbb{S}_{\mathrm{CHD}}^{0}$ be given by ((6)). If we set $\gamma=\pi / 2$ and $\omega_{1}=-z^{3}$ then by shear construction of Clunie and Sheil-Small [2], we have

$$
\begin{aligned}
h_{\gamma}(z)= & \frac{1}{6} \log (1+z)-\frac{i}{4} \log \left(\frac{1+i z}{1-i z}\right) \\
& +\frac{1}{4} \log \left(1+z^{2}\right)-\frac{1}{3} \log \left(1-z+z^{2}\right), \\
g_{\gamma}(z)= & \frac{1}{6} \log (1+z)+\frac{i}{4} \log \left(\frac{1+i z}{1-i z}\right) \\
& +\frac{1}{4} \log \left(1+z^{2}\right)-\frac{1}{3} \log \left(1-z+z^{2}\right) .
\end{aligned}
$$

Recall that, if $f=h+\bar{g} \in \mathbb{S}_{H}$, then

$$
P_{\delta} * f=\frac{\delta z h^{\prime}+h}{1+\delta}+\overline{\frac{\delta z g^{\prime} g}{1+\delta}} .
$$




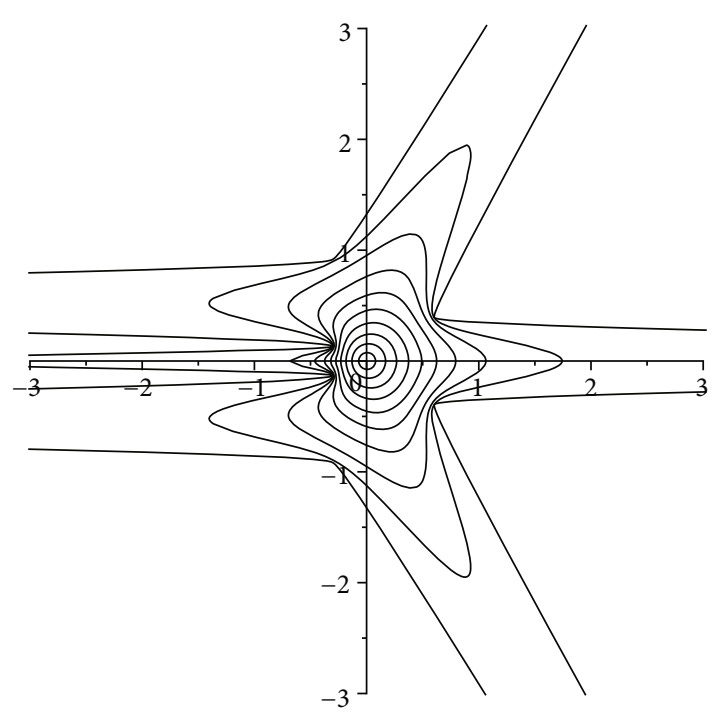

FIGURE 7: Image of $F_{-1 / 3} * f_{2 \Pi / 3}$.

So, we have

$$
\begin{aligned}
& P_{\delta} * f_{\gamma}=\frac{1}{1+\delta}\left[\delta z h_{\gamma}^{\prime}(z)+h_{\gamma}(z)\right]+\frac{1}{1+\delta} \overline{\left[\delta z g_{\gamma}^{\prime}(z) g_{\gamma}(z)\right]} \\
& =\frac{1}{1+\delta}\left[\frac{\delta z}{\left(1+z^{3}\right)\left(1+z^{2}\right)}+\frac{1}{6} \log (1+z)-\frac{i}{4} \log \right. \\
& \left.\cdot\left(\frac{1+i z}{1-i z}\right)+\frac{1}{4} \log \left(1+z^{2}\right)-\frac{1}{3} \log \left(1-z+z^{2}\right)\right] \\
& +\frac{1}{1+\delta}\left[\frac{\delta z^{4}}{\left(1+z^{3}\right)\left(1+z^{2}\right)} \frac{1}{6} \log (1+z) \frac{i}{4} \log \left(\frac{1+i z}{1 i z}\right)\right. \\
& \overline{\frac{1}{4} \log \left(1+z^{2}\right)+\frac{1}{3} \log \left(1 z+z^{2}\right)} \\
& =\operatorname{Re}\left\{\frac{1}{1+\delta}\left[\frac{\delta z\left(1-z^{3}\right)}{\left(1+z^{3}\right)\left(1+z^{2}\right)}-\frac{i}{2} \log \left(\frac{1+i z}{1-i z}\right)\right]\right\} \\
& +i \operatorname{Im}\left\{\frac { 1 } { 1 + \delta } \left[\frac{\delta z}{1+z^{2}}+\frac{1}{3} \log (1+z)\right.\right. \\
& \left.\left.+\frac{1}{2} \log \left(1+z^{2}\right)-\frac{2}{3} \log \left(1-z+z^{2}\right)\right]\right\} \text {. }
\end{aligned}
$$

Now, in view of Theorem 11, if we set the parameter $\delta=2 / 3$, then $P_{\delta} * f_{\gamma}$ is univalent and CHD. Also, if we choose $\delta=3$ 14 , then $P_{\delta} * f_{\gamma}$ is not guaranteed to be univalent. The images of $|z|=r<1$ under $f_{\Pi / 2}, P_{2 / 3}, P_{2 / 3} * f_{\Pi / 2}$ and $P_{3 / 4} * f_{\Pi / 2}$ are shown in Figures 1-4.

Example 15. Suppose $f_{\gamma}=h_{\gamma}+\overline{g_{\gamma}} \in \mathbb{S}_{\mathrm{CHD}}^{0}$ be given by (6). If we set $\gamma=2 \pi / 3$ and $\omega_{2}=z^{4}$, then calculations lead to

$$
\begin{aligned}
h_{\gamma}(z)= & \frac{1}{12} \log (1+z)+\frac{1}{4} \log \left(\frac{1+z^{2}}{1-z}\right) \\
& -\frac{1}{6} \log \left(1-z+z^{2}\right)-\frac{i \sqrt{3}}{6} \log \left(\frac{1+z e^{(2 \pi / 3) i}}{1+z e^{(-2 \pi / 3) i}}\right),
\end{aligned}
$$

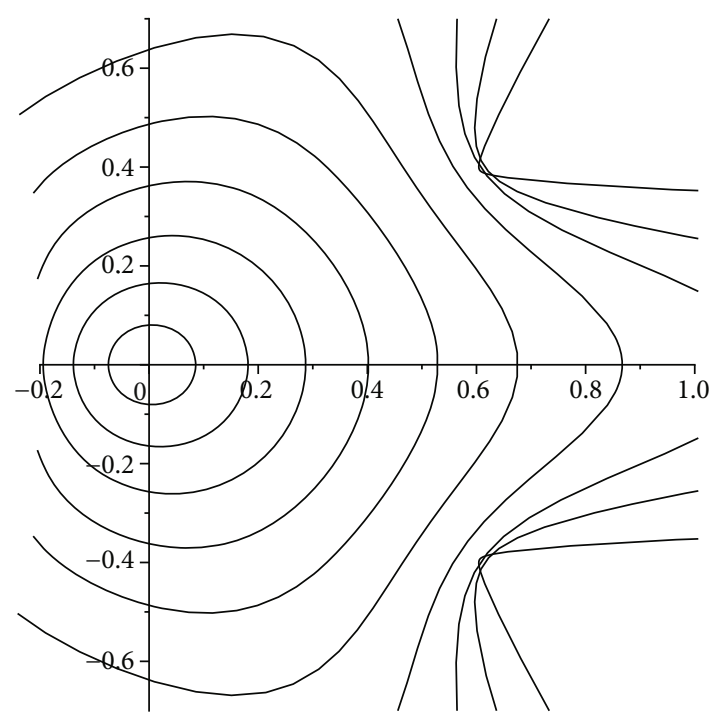

Figure 8: Image of $F_{-1 / 4} * f_{2 \Pi / 3}$.

$$
\begin{aligned}
g_{\gamma}(z)= & \frac{1}{12} \log (1+z)+\frac{1}{4} \log \left(\frac{1+z^{2}}{1-z}\right) \\
& -\frac{1}{6} \log \left(1-z+z^{2}\right)+\frac{i \sqrt{3}}{6} \log \left(\frac{1+z e^{(2 \pi / 3) i}}{1+z e^{(-2 \pi / 3) i}}\right) .
\end{aligned}
$$

If $f=h+\bar{g} \in \mathbb{S}_{H}$, then

$$
F_{\mathfrak{a}} * f=\frac{1}{2}\left[\frac{(1+\mathfrak{a}) z h^{\prime}}{1-\mathfrak{a}}+h\right]+\frac{1}{2} \overline{\left[\frac{(1+\mathfrak{a}) z g^{\prime}}{1 \mathfrak{a}} g\right]} .
$$

So, we have

$$
\begin{aligned}
F_{\mathfrak{a}} * f_{\gamma}= & \left.\frac{1}{2}\left[\frac{(1+\mathfrak{a}) z h_{\gamma}^{\prime}}{1-\mathfrak{a}}+h_{\gamma}\right]+\frac{1}{2} \overline{\frac{(1+\mathfrak{a}) z g_{\gamma}^{\prime}}{1 \mathfrak{a}}} g_{\gamma}\right] \\
= & \frac{1}{2}\left[\frac{(1+\mathfrak{a}) z}{(1-\mathfrak{a})\left(1-z+z^{2}\right)\left(1-z^{4}\right)}+\frac{1}{12} \log (1+z)\right. \\
& +\frac{1}{4} \log \left(\frac{1+z^{2}}{1-z}\right)-\frac{1}{6} \log \left(1-z+z^{2}\right) \\
& \left.-\frac{i \sqrt{3}}{6} \log \left(\frac{1+z e^{(2 \pi / 3) i}}{1+z e^{(-2 \pi / 3) i}}\right)\right] \\
& +\frac{1}{2}\left[\frac{(1+\mathfrak{a}) z^{5}}{(1 \mathfrak{a})\left(1 z+z^{2}\right)\left(1 z^{4}\right) \frac{1}{12} \log (1+z) \frac{1}{4} \log \left(\frac{1+z^{2}}{1 z}\right)}\right. \\
& \left.+\frac{1}{6} \log \left(1 z+z^{2}\right) \frac{i \sqrt{3}}{6} \log \left(\frac{1+z e^{(2 \pi / 3) i}}{1+z e^{(2 \pi / 3) i}}\right)\right] \\
= & \operatorname{Re}\left\{\frac { 1 } { 2 } \left[\frac{(1+\mathfrak{a}) z\left(1+z^{4}\right)}{(1-\mathfrak{a})\left(1-z+z^{2}\right)\left(1-z^{4}\right)}\right.\right. \\
& \left.\left.-\frac{i \sqrt{3}}{3} \log \left(\frac{1+z e^{(2 \pi / 3) i}}{1+z e^{(-2 \pi / 3) i}}\right)\right]\right\} \\
& +i \operatorname{Im}\left\{\frac { 1 } { 2 } \left[\frac{(1+\mathfrak{a}) z}{(1-\mathfrak{a})\left(1-z+z^{2}\right)}+\frac{1}{6} \log (1+z)\right.\right. \\
& \left.\left.+\frac{1}{2} \log \left(\frac{1+z^{2}}{1-z}\right)-\frac{1}{3} \log \left(1-z+z^{2}\right)\right]\right\} .
\end{aligned}
$$


Now, if we set the parameter $\mathfrak{a}=-1 / 3$, in view of Theorem $11, F_{\mathfrak{a}} * f_{\gamma}$ is univalent and CHD. If we choose $\mathfrak{a}=-1 / 4$, then $F_{\mathfrak{a}} * f_{\gamma}$ is not guaranteed to be univalent (see Figures 5-8).

\section{Data Availability}

No data were used to support this study.

\section{Conflicts of Interest}

The authors declare that there is no conflict of interest.

\section{References}

[1] H. Lewy, "On the non-vanishing of the Jacobian in certain one-to-one mappings," Bulletin of the American Mathematical Society, vol. 42, no. 10, pp. 689-693, 1936.

[2] J. Clunie and T. Sheil-Small, "Harmonic univalent functions," Annales Academioe Scientiarum Fennica Mathematica, vol. 9, pp. 3-25, 1984.

[3] P. Duren, Harmonic Mapping in the Plane, Cambridge University Press, Cambridge, 2004.

[4] M. Dorff, "Convolutions of planar harmonic convex mappings," Complex Variables, Theory and Application: An International Journal, vol. 45, no. 3, pp. 263-271, 2001.

[5] M. Dorff, M. Nowak, and M. Woloszkiewicz, "Convolutions of harmonic convex mappings," Complex Variables and Elliptic Equations, vol. 57, no. 5, pp. 489-503, 2012.

[6] M. Dorff, S. G. Hamidi, J. M. Jahangiri, and E. Yasar, "Convolutions of planar harmonic strip mappings," Complex Variables and Elliptic Equations, pp. 1-18, 2020.

[7] Y. Li and Z. Liu, "Convolutions of harmonic right half-plane mappings," Open Mathematics, vol. 14, no. 1, pp. 789-800, 2016.

[8] Z. Liu and Y. Li, "Corrigendum to "the properties of a new subclass of harmonic univalent mappings"," Abstract and Applied Analysis, vol. 2015, Article ID 794108, 2015.

[9] Z. Liu, Y. Jiang, and Y. Sun, "Convolutions of harmonic halfplane mappings with harmonic vertical strip mappings," Filomat, vol. 31, no. 7, pp. 1843-1856, 2017.

[10] Z. Liu, Z. Wang, A. Rasila, and Y. Jiang, "Convolutions of harmonic right half-plane mappings with harmonic strip mappings," Bulletin of the Malaysian Mathematical Sciences Society, vol. 42, no. 3, pp. 1199-1212, 2019.

[11] C. Singla, S. Gupta, and S. Singh, "An alternative approach to convolutions of harmonic mappings," in Mathematical Analysis and Computing. ICMAC 2019, R. N. Mohapatra, S. Yugesh, G. Kalpana, and C. Kalaivani, Eds., vol. 344 of Springer Proceedings in Mathematics \& Statistics, pp. 623-633, Springer, Singapore, 2021.

[12] E. Yasar, "On convolutions of slanted half-plane mappings," Journal of Taibah University for Science, vol. 15, no. 1, pp. 71-76, 2021.

[13] R. Kumar, S. Gupta, S. Singh, and M. Dorff, "An application of Cohn's rule to convolutions of univalent harmonic mapping," Rocky Mountain Journal of Mathematics, vol. 46, no. 2, pp. 559-570, 2016.

[14] Z. Wang, Z. Liu, and Y. Li, "On convolutions of harmonic univalent mappings convex in the direction of the real axis," Journal of Applied Analysis \& Computation, vol. 6, no. 1, pp. 145-155, 2016.
[15] E. Yasar and O. Ozdemir, "Convolutions of a subclass of harmonic univalent mappings," TWMS Journal of Applied and Engineering Mathematics, vol. 10, no. 2, pp. 353-359, 2020.

[16] C. Pommerenke, "On starlike and close-to-convex functions," Proceedings of the London Mathematical Society, vol. 13, pp. 290-304, 1963.

[17] P. Duren, Univalent functions, Springer-Verlag, New York, 1983.

[18] Q. I. Rahman and G. Schmeisser, Analytic Theory of Polynomials, Oxford University Press, Oxford, 2002.

[19] M. Marden, Geometry of Polynomials, American Mathematical Society, 1966. 